\title{
Contrast agents for molecular photoacoustic imaging
}

\section{Judith Weber ${ }^{1,2}$, Paul C Beard ${ }^{3^{*}}$ and Sarah E Bohndiek ${ }^{1,2^{\star}}$}

${ }^{1}$ Department of Physics, University of Cambridge, Cambridge, U.K.

${ }^{2}$ Cancer Research UK Cambridge Institute, University of Cambridge, Cambridge, U.K.

${ }^{3}$ Department of Medical Physics and Biomedical Engineering, University College London, London, U.K.

* Corresponding Author information:

Professor Paul C Beard: Professor of Biomedical Photoacoustics. Department of Medical Physics and Bioengineering, University College London, Malet Place Engineering Building, London, WC1E 6BT, U.K.

Phone: +44 207679 0290; Fax: +44 207679 0255; Email: paul.beard@ucl.ac.uk.

Dr Sarah E Bohndiek: University Lecturer in Biomedical Physics. Department of Physics, JJ Thomson Avenue, Cambridge, CB3 OHE, U.K. and Junior Group Leader, Cancer Research UK Cambridge Institute, Li Ka Shing Centre, Robinson Way, Cambridge, CB2 ORE, U.K.

Phone: +44 1223 337267; Fax: +44 1223 337000; Email: seb53@cam.ac.uk.

\begin{abstract}
Photoacoustic imaging (PAI) is an emerging tool that bridges the traditional depth limits of ballistic optical imaging and the resolution limits of diffuse optical imaging. Using the acoustic waves generated in response to the absorption of pulsed laser light, it provides non-invasive images of absorbed optical energy density at depths of several centimeters with a resolution of $\sim 100 \mu \mathrm{m}$. This versatile and scalable imaging modality has now shown potential for molecular imaging, which enables visualization of biological processes non-invasively using systemically introduced contrast agents. Understanding the relative merits of the vast range of contrast agents reported, from small molecule dyes, through gold and carbon nanostructures, to liposome encapsulations, is a significant challenge. Here, we critically review the physical, chemical and biochemical characteristics of the existing photoacoustic contrast agents, highlighting key applications and present challenges for molecular PAI.
\end{abstract}




\section{Introduction}

Molecular imaging aims to visualize and quantify biological processes at the molecular and cellular level in a non-invasive manner, providing an opportunity to detect, stage, predict and monitor the development of disease. As early as the $17^{\text {th }}$ century, Anton van Leeuwenhoek and Robert Hooke used light to visualize cells. Light experiences significant scattering as it passes through tissue, which requires an inherent tradeoff between depth of penetration and spatial resolution. Microscopy and other techniques that utilize unscattered photons provide high resolution $(\sim 1 \mu \mathrm{m})$ images but only to a maximum depth of $\sim 1 \mathrm{~mm}$ in most tissues; diffuse optical techniques such as fluorescence and near infrared optical tomography that exploit multiply scattered photons can reach depths of several $\mathrm{cm}$ provided spatial resolution is sacrificed $(>1 \mathrm{~mm}){ }^{1}$

Photoacoustic imaging (PAI) is an emerging method that combines the high contrast of optical imaging with the high spatial resolution of ultrasound. ${ }^{2-5}$ Tissue heating due to the absorption of short laser pulses by endogenous chromophores or exogenous contrast agents produces broadband acoustic waves at $\mathrm{MHz}$ frequencies. These photoacoustic waves can be detected by ultrasound transducers at the tissue surface and reconstructed to form an image of the absorbed optical energy distribution ${ }^{2}$. Supplementary Fig. 1 and Note 1 illustrate this process and explain the requirements for the image to represent absorbed optical energy. Since sound waves are less scattered in tissue than photons, PAI can push the traditional limitations of optical imaging. For example, when operating in the "nearinfrared (NIR) window' (620 - $950 \mathrm{~nm}$ ), tomographic PAI can reach a penetration depth of several centimeters with a resolution of the order of a few $100 \mu \mathrm{m}$, scaling to $<100 \mu \mathrm{m}$ resolution for shallower $(<1 \mathrm{~cm})$ penetration depths. ${ }^{3}$

PAI is currently one of the fastest-growing molecular imaging techniques. It has rapidly been adopted for preclinical in vivo imaging in small animals for a range of disease indications. The prevailing application is in cancer research, where PAI has been explored for: primary tumor detection and molecular characterization ${ }^{6,7}$; therapeutic monitoring ${ }^{8}$; as well as for the identification and assessment of metastatic lymph nodes ${ }^{9}$.

This review highlights the latest advances in exogenous contrast agents for molecular PAI. We introduce the considerations for in vivo molecular PAI, detail the physical, chemical and biochemical characteristics of available contrast agents, and provide an overview of the strengths and weaknesses of each approach. Given the widespread use of this technique in cancer research, we draw on this field for key examples of the application of molecular PAI. 


\section{Sources of endogenous contrast in PAI}

While the focus of this review is exogenous contrast agents, it is important to appreciate the capabilities of, and challenges presented by, background endogenous PA signals when performing molecular PAI.

\section{Intrinsic chromophores}

Endogenous PAI provides structural and functional information through light absorption by hemoglobin, lipids, water and melanin (Fig. 1a). Hemoglobin, which delivers oxygen throughout the body, experiences structural and electronic modifications upon oxygen binding (Fig. 1b). The resulting changes in the absorption spectrum (Fig. 1a) offer the prospect of measuring total hemoglobin concentration $\left(\mathrm{Hb}_{\text {total }}\right)$ and oxygen saturation $\left(\mathrm{sO}_{2}\right)$, important for studying tumor angiogenesis (Fig. 1c).$^{10,8}$ PAI can also map lipid distribution in vivo ${ }^{11}$ based on the absorption peaks at 930 and 1,210 nm (second overtone of abundant $\mathrm{C}-\mathrm{H}$ bonds), while water can be identified based on the absorption peak at 975 $\mathrm{nm} .{ }^{12}$ Melanin is a ubiquitous natural pigment in skin, hair and eyes, with intense optical absorption, enabling sensitive characterization of primary melanoma ${ }^{13,14}$ and metastatic melanoma cells ${ }^{15}$. Intrinsic chromophores therefore provide access to only a limited range of biological processes and when performing molecular PAI, they represent the background absorption that must be overcome by contrast agent design.

\section{Genetically encoded chromophores}

Most cells in the body are invisible to PAI in the NIR window. Reporter gene imaging enables direct visualization of the location and level of gene expression, promoter activity and protein-protein interaction in vivo. ${ }^{16}$ Under the control of the promoter of the gene of interest, a reporter gene expresses proteins that either provide PA contrast directly or create a product that yields PA contrast (Fig. 2a).

For example, the genetically encoded family of green fluorescent proteins (GFPs) ${ }^{17}$ can provide direct contrast. The GFP family is, however, too blue-shifted for in vivo applications beyond virtually transparent organisms ${ }^{17}$ due to strong background absorption by endogenous chromophores at visible wavelengths (Fig. 1a). Unfortunately, the GFP-like red-shifted fluorescent proteins exhibit limited PA signal generation efficiency and photostability. ${ }^{18}$ The development of phytochrome-based NIR fluorescent proteins have begun to overcome these limitations ${ }^{19,20}$. Phytochromes are photosensory receptors that absorb light when they covalently bind a linear tetrapyrrole chromophore, such as biliverdin. Since biliverdin is an intermediate of normal mammalian heme metabolism, phytochromes are promising candidates as PAI genetic reporters for in vivo imaging. The first example in this class was iRFP, which can be excited at $\sim 690 \mathrm{~nm}$ and shows good photo- and thermodynamic stability in vivo. ${ }^{20}$ More recently, differential imaging of the on / off states in the reversibly switchable phytochrome BphP1 has been demonstrated. Excitation of the biliverdin-BphP1 complex with light in the range of 730-790 nm triggers the bound biliverdin to switch from trans to cis conformation (Fig. 2b), causing a change in the absorption spectrum towards $630-690 \mathrm{~nm} .{ }^{21}$ The photoconversion was shown to reduce the impact of 
background signals (Fig. 2c), improve detection sensitivity and increase penetration depth. Reversibly switchable phytochromes may therefore play a significant role in future studies.

More widely explored transgenic approaches for PAl are based on enzymatic reporter genes. Here, the genetic product is an enzyme that produces a chromophore or its precursor. For instance, LacZ encodes the enzyme $\beta$-galactosidase, which metabolizes the transparent precursor substrate X-gal to produce a stable, dark-blue product. ${ }^{22}$ Dualwavelength PAl can detect tumor cells expressing lacZ up to $5 \mathrm{~cm}$ depth with high resolution. ${ }^{22}$ The lacZ-reporter gene system is limited, however, as X-gal must be locally administered and the metabolic product shows poor clearance. The tyrosinase-reporter gene system (Fig. 2d,e) introduces the key enzyme in melanin synthesis into non-melanogenic cells, hence does not require an exogenously administered substrate. ${ }^{23}$ Although the relatively featureless absorption spectrum of melanin (Fig. 1a) could make it hard to distinguish from intrinsic signals, in vitro and in vivo PAl of tyrosinase-expressing cells has shown high sensitivity ${ }^{23,24}$ and inducible systems can address potential toxicity concerns. ${ }^{25}$ Despite their potential for signal amplification, enzymatic reporter systems are generally less desirable due to their dependence on substrate availability, which limits quantification.

Reporter genes are suitable for applications where the cell or biological process of interest does not provide inherent PA contrast but use of an exogenously applied contrast agent is undesirable, for example, requiring repeated injections in longitudinal studies of tumor development. They are, however, subject to the same considerations as exogenous contrast agents in terms of desired photophysical properties, as discussed in the following sections. 


\section{Exogenous contrast agent considerations for molecular PAI}

To perform molecular imaging, a contrast agent (Fig. 3) must be composed of a signaling compound, which produces the signal for imaging, and a targeting moiety that enables the readout of a specific biological entity or process.

\section{Photophysical properties: signaling compounds}

The ideal signaling compound needs to exhibit the following characteristics: high molar extinction coefficient (Fig. 4), to maximize the amount of light absorbed; sharply peaked, characteristic absorption spectrum, to ensure unambiguous identification by spectral unmixing even at low molar concentrations (Supplementary Note 1); peak absorption in the NIR window $(620-950 \mathrm{~nm})$, to maximize penetration depth by avoiding the strong absorption of intrinsic chromophores (Fig. 1a); high photostability, to ensure that spectral features are not changed by light irradiation; low quantum yield, to maximize the nonradiative conversion of light energy to heat; and efficient conversion of heat energy ${ }^{3}$ to produce acoustic waves.

\section{Biological properties: targeting and biocompatibility}

The targeted contrast agent must overcome circulatory and cellular barriers (Supplementary Fig. 2). Contrast agents will first interact with biomolecules in the bloodstream, ${ }^{26,27}$ where adsorption of biomolecules onto the surface will impact both immune responses and target binding. ${ }^{27,28}$ This needs characterization and consideration when interpreting biological results. Furthermore, to reach their target, these agents typically need to pass out of the circulatory system. To extravasate, a maximum size limit of typically $<10$ $\mathrm{nm}$ is necessary, although for applications in cancer, contrast agents of up to $100 \mathrm{~nm}$ have been found to pass through the leaky vessels found in tumors ${ }^{29}$. Once in the tissue interstitium, passing the cellular barrier can be achieved by active targeting, for example to cell surface receptors ${ }^{30}$, transporters ${ }^{31}$, or metabolic enzymes ${ }^{32}$, or by relying on passive uptake through diffusion or endocytosis.

To facilitate active targeting, the biological target should be (over)expressed at an early stage of the disease and found at low levels in off-target tissues. Structures that can be used to achieve active targeting include small molecules, peptides and adhirons ${ }^{33}$, affibodies $^{34}$, aptamers ${ }^{35}$ and proteins (Fig. 3). In particular, consideration must be given to biocompatibility, which refers here to minimizing toxicity (e.g. based on administration route and dose) and immune response (e.g. by reducing accumulation in non-targeted tissue), as well as optimizing elimination from the circulatory system and target tissue penetration.

For example, combining small molecules such as sugars with small molecule dyes results in a small $(<1 \mathrm{~nm})$ contrast agent that can easily pass physiological barriers and clear rapidly through the kidneys, which reduces off-target accumulation and optimizes biocompatibility. Conversely, although proteins (e.g. monoclonal antibodies) show high target affinity and specificity, and thus display little cross-reactivity with healthy tissues, their larger size results in poorer tissue penetration and clearance, and immunogenicity is possible, resulting in poorer biocompatibility. Antibody fragments, including dia- and nano- 
bodies, are now being developed to address these challenges, since they are smaller in size but retain high affinity and selectivity. ${ }^{34,36}$

For cancer research, the hyperpermeability of tumor vasculature combined with the lack of an intact lymphatic drainage may lead to 'enhanced permeability and retention' (EPR) of contrast agents of up to $\sim 100 \mathrm{~nm}$ inside the tumor tissue without the need for targeting ligands. ${ }^{37}$ 'Passive' targeting based on the EPR has been demonstrated extensively in mice (Supplementary Tables 1-4) and recently the first studies have begun to emerge in humans ${ }^{38}$. Larger entities $(>100 \mathrm{~nm}$ ) are typically not accumulated and will be cleared through the reticuloendothelial system. ${ }^{29}$ Passive targeting confounds attempts to perform targeted molecular imaging, as uptake is not specific to a biological process hence should be avoided as far as possible by contrast agent design. 


\section{Signaling compounds for molecular PAI}

Signaling compounds fall into three broad classes: small molecule dyes; metallic or semiconducting nanostructures; and organic nanostructures (Fig. 3). Supplementary Tables 1-4 summarize PAI studies using different classes of compounds. Figure 4 enables direct comparison of two critical parameters for the ideal signaling compound: the specific extinction coefficients and peak absorption wavelengths for the different classes.

\section{Small molecule dyes}

Small molecule NIR dyes ${ }^{39}$ (Supplementary Table 1) are generally commercially available. The chemical basis of these dyes is a series of conjugated double-bonds and/or (mostly aromatic) ring systems. In these highly conjugated structures, electrons are delocalized, meaning less energy is needed for excitation. The well-defined energy gap between ground and excited states results in a distinct absorption peak (Supplementary Fig. 3). Relaxation of the excited electrons can occur in radiative (fluorescence or bioluminescence) or non-radiative processes, dependent on the lifetime and electronic configuration of the excited states (Fig. 5a). ${ }^{40}$ For PAI, a large non-radiative relaxation is required so the quantum yield (referring to fluorescence emission) should be as low as possible.

The first main class of NIR dyes used for PAl is the heptamethine cyanine dyes comprising two aromatic nitrogen-containing heterocycles linked by a heptamethine chain. ${ }^{41,42}$ An example is indocyanine green (ICG), an FDA approved dye with low toxicity. In aqueous solution, ICG has an absorption maximum at $780 \mathrm{~nm}$ and a relatively low quantum yield for fluorescence $(0.027) .{ }^{43}$ Due to its inherent hydrophobicity, ICG exhibits high plasma protein binding and is used clinically as a blood flow contrast agent. ${ }^{44}$ ICG was rapidly applied in cancer research studies using PAI for identification of the closest 'sentinel' lymph node (SLN) in the lymphatic drainage from a tumor ${ }^{45}$, which is crucial for cancer staging. However, the hydrophobicity of ICG (and other small molecule dyes) can result in significant concentration- and environment- dependent changes in optical properties (Fig. 5b) ${ }^{46}$. Also, functionalization and targeting chemistry of ICG is challenging; unmodified ICG is therefore not ideal for molecular $\mathrm{PAI}^{47,48}$ and alternative heptamethine cyanine dyes have been exploited for this purpose (Supplementary Table 1).

Squaraines are the second main class of NIR dyes that could be employed for molecular PAI. They are characterized by an electron deficient central 4-membered ring often conjugated to two electron-donating groups to generate a donor-acceptor-donor form, which increases the delocalization of the electrons. The optical properties can be tuned by varying donor strengths to produce sharp absorption bands in the NIR range. ${ }^{49}$ Despite good optical properties and photostability, squaraines are often limited by insolubility, aggregation and chemical reactivity. ${ }^{50,51}$ In PAl they have only been used in conjunction with phospholipids, albumin or polymers owing to poor solubility. ${ }^{52-54}$ Additional dye classes with fewer examples in PAI are rhodamines and azo dyes. Rhodamines are characterized by a xanthene core unit with diakylamino groups and have high photostability ${ }^{55}$. Azo dyes Methylene Blue (MB, $664 \mathrm{~nm}$ ) and Evans Blue (EB, $620 \mathrm{~nm}$ ) have been used for PAI, but are limited by relatively blue shifted absorption peaks ${ }^{56,57}$ and potential to cause photodamage under intense irradiation. ${ }^{58}$ 
Challenges associated with the use of small molecule dyes include: low molar extinction coefficient $\left(<3 \times 10^{5} \mathrm{M}^{-1} \mathrm{~cm}^{-1}\right)$; low solubility; and tendency to aggregate. It is possible to address some of these challenges chemically, by attaching hydrophilic groups, introducing triplet-state quenchers ${ }^{59}$ and integrating stabilizing groups ${ }^{60}$. Additionally, variations in the chemical design, such as varying the size of the conjugated system, attaching electron donating / withdrawing groups, allow tailoring of the spectral range. Their chemical flexibility also facilitates the creation of 'smart' contrast agents (Section 'Emerging concepts in molecular PAl'), which can in part compensate for their low molar extinction coefficient by minimizing the influence of the background signal from endogenous chromophores.

Most small molecule dyes must be actively targeted to gain specificity. A common target for detection and characterization of solid tumors in preclinical research is the cell surface epidermal growth factor receptor (EGFR), which is frequently overexpressed. Hudson et al explored the potential of molecular PAl for detecting EGFR-expressing tumors in a mouse model of pancreatic cancer (depths $>5 \mathrm{~mm}$ ) by conjugating the epidermal growth factor (EGF) protein to the rhodamine NIR dye CF-750 (Fig. 5c). ${ }^{61}$ The absorption spectrum of CF-750 was not significantly altered by the bioconjugation to EGF and the probe signal could be clearly distinguished from background by multi-wavelength PAI, providing a noninvasive readout of EGFR expression. Importantly, there was no noted toxicity.

In spite of their photophysical limitations, small molecule dyes remain good candidates for molecular PAI in the preclinical study of extravascular targets due to their small size and favorable biocompatibility.

\section{Nanostructures}

Nanoparticles (NPs) embody the largest class of contrast agents for molecular PAI. They possess high chemical, physical and biochemical flexibility, as well as significantly increased molar extinction coefficients compared to small molecule dyes. NPs exist in different materials, shapes and sizes $(<1000 \mathrm{~nm}$, usually $\sim 10-100 \mathrm{~nm})$, and have tunable surface properties, reactivity and optical characteristics. Their large surface area means a large number of targeting moieties can be attached per particle, increasing target-binding probability. However, due to their larger size, NPs often accumulate in the reticuloendothelial system for long time periods, yielding poorer biocompatibility. Furthermore, the reproducibility, purification, and quantification of homogeneous, well-defined NPs remains challenging. ${ }^{62}$ Details of common synthesis routes for all nanostructures and nanomaterials demonstrated for PAI can be found in Supplementary Notes 2 and 3.

\section{Gold}

The main focus of research into metallic nanoparticles for molecular PAI has been on gold, owing to its unique optical and physicochemical properties, relatively inert nature, and prior use in humans. In addition, gold forms strong gold-thiolate bonds that enable covalent surface modifications for optimizing biocompatibility (e.g. poly(ethylene glycol) (PEG) functionalization $)^{63}$, stability (e.g. silica encapsulation) ${ }^{64}$ and active targeting.

The optical properties of gold nanoparticles (GNPs) arise from the 'localized surface plasmon resonance' (LSPR) phenomenon. The conduction electrons of a GNP oscillate 
relative to the core when exposed to light of an appropriate wavelength (Fig. 6a). This state is referred to as plasmon. ${ }^{65}$ Plasmons convert a significant part of the oscillation energy into heat detectable with PAI. The key advantage of GNPs for PAI signal generation is that they essentially act as a macroscopic dipole, leading to an extinction coefficient several orders of magnitude higher than small molecule dyes (extinction scales $\sim$ volume ${ }^{\wedge} 3$ ). ${ }^{66}$ GNPs show the highest extinction coefficient in the NIR range and high PA conversion efficiency.

The frequency of the LSPR determines the peak absorption wavelength of the GNP, which can be tuned based on: surface-to-volume ratio; polarization modes; edge / vertex number and 'sharpness' of the GNP. Therefore, in addition to spheres, a wide range of GNP shapes, including stars, tripods, plates and prisms have emerged (Supplementary Table 2). A key example of tuning is found in gold nanorods (GNRs), whose intense and narrow LSPR absorption bands in the NIR are tuned by varying their aspect ratio (Fig. 6b). GNRs are commonly encapsulated in silica, which reduces gold interfacial thermal resistance with the solvent, which improves PA conversion efficiency ${ }^{64,67}$ and photostability. ${ }^{68}$ Another example is the use of hollow gold nanostructures, such as gold nanocages (GNCs) and gold nanoshells (GNSs) that can also encapsulate cargo such as drugs within their porous walls. The LSPR frequency can be conveniently tuned not only based on size but also on shell thickness (thinner $=$ red shift) $\left(\right.$ Fig. $6 \mathrm{c}$ ) $\cdot{ }^{69,70}$ These simple methods for tuning the characteristic spectral shape and peak wavelength are highly advantageous for molecular PAI.

In diseases with a leaky vasculature, like cancer, GNPs can be accumulated via the EPR effect. ${ }^{71}$ Actively targeting the angiogenic tumor vasculature (in contrast to quiescent surrounding vessels) provides a tumor specific readout that circumvents the physiological barrier of extravasation into the tissue. The tripeptide arginine-glycine-aspartic acid (RGD) and its derivatives are widely used to target the $\alpha_{\mathrm{v}} \beta_{3}$ integrin on angiogenic vessels. All studies to date used a bifunctional PEG linker to conjugate the GNP with the RGD derivative (Supplementary Table 2). Studies with larger RGD-modified GNPs suffer from high accumulation in the reticuloendothelial system and slow tumor accumulation of at least 6 h. ${ }^{30,72}$ This is unfavorable in terms of biocompatibility, as it renders the reticuloendothelial system organs prone to oxidative damage via free radical formation. ${ }^{73}$ These limitations were recently overcome using small gold tripods $(<20 \mathrm{~nm})$ conjugated to the RGD derivative cyclic Arg-Gly-Asp-D-Phe-Cys via a PEG linker (Fig. 6d, e). ${ }^{74}$ PAI results were verified with ${ }^{64} \mathrm{Cu} P E T$. Encouragingly, RGD-gold tripods showed rapid accumulation in a U87MG glioma within 2 hours without systemic toxicity.

Targeting GNRs to extravascular cell surface receptors has been demonstrated using antibody conjugation. Due to their relatively narrow spectrum, GNRs tuned to absorb at a range of different peak wavelengths can be conjugated to multiple targets, which has enabled multiplexed characterization of tumor EGFR and human epidermal growth factor receptor 2 (HER2) expression ${ }^{7}$ allowing characterization of heterogeneous tumors and specific tracking of combination therapies. It remains to be seen whether the use of GNPs in reaching extravascular targets generalizes across disease types and to applications in healthy tissue with intact vasculature.

GNPs possess the most flexible photophysical properties of the compounds reviewed. Easy and stable functionalization of GNPs can be made via gold-thiolate bonds, suitable for attachment of a range of targeting moieties and further signaling compounds for multimodal imaging. Their associated spectral flexibility makes GNPs highly suitable for multiplexed imaging of different targets within the same tissue. Nonetheless, interaction of 
the GNPs reviewed here within the vascular and tissue compartments depends on their size, shape, charge and synthesis route. Biocompatibility in vivo therefore needs to be more systematically addressed through longer term toxicity studies for individual nanostructures.

\section{Carbon}

Carbon forms different allotropic structures: carbon nanotubes, graphene-based nanomaterials and nanodiamonds (Supplementary Table 3). All three classes can be synthesized to possess an intrinsic absorption in the NIR range (Fig. 7a,b, Supplementary Fig. 4 and Note 2). Despite possessing a lower molar extinction coefficient than gold, carbon nanoparticles (CNPs) hold significant potential for molecular PAI due to their flexibility of synthesis and functionalization. Of particular interest is the wide variety of covalent ${ }^{75-78}$ as well as non-covalent modifications with small molecules $\left(\right.$ dyes $^{79}$, drugs ${ }^{80}$, surfactants ${ }^{81}$ ), polymers $^{79}$ and biomolecules ${ }^{82}$ that can be used for: targeting; increased solubility and stability $^{83}$; improved signaling properties ${ }^{84}$ or increased specificity ${ }^{79}$. The absorption spectra of CNPs tend to be relatively broad in the NIR range, presenting a challenge for spectral unmixing in molecular PAI, but the attachment of NIR dyes can circumvent this limitation (Supplementary Fig. 5).

The majority of molecular PAI studies using carbon have been performed using single-walled carbon nanotubes (SWNT). SWNTs can be visualized as cylindrically rolled-up graphene sheets with diameters of $1-3 \mathrm{~nm}$ and lengths up to centimeters (Fig. 7a). ${ }^{85}$ Semiconducting SWNT can exhibit numerous optical absorption peaks (Fig. 7a,b). The absorption peaks are defined by narrow energy ranges where many electronic states are available (referred to as van Hoff singularities, Fig. 7b). The electronic, and thus optical, properties are tunable by changing the tube structure, for example, diameter and chirality. The band gap generally varies inversely with the diameter, but many open questions remain regarding the complex photophysics of SWNTs. ${ }^{86,87}$

Molecular targeting of the $\alpha_{v} \beta_{3}$ integrin for angiogenesis imaging has also been achieved in multiple studies of CNPs using RGD-peptides attached to SWNT. ${ }^{79,88-90}$ De la Zerda et al conjugated RGD peptides through PEG grafted phospholipids to SWNT. In vivo evaluation found a strong PAI signal in the tumor for SWNT-RGD compared to only weak signals (via passive EPR uptake) for non-targeted SWNTs (Fig. 7c). ${ }^{88}$ Attachment of NIR dyes to the surface of the nanotubes achieved substantial PA contrast and sub-nanomolar sensitivities in mice compared to plain SWNT. ${ }^{79,90}$ The underlying mechanism of the enhancement was not established, but quenching effects between the dyes and SWNT may play a part. It should be noted that photobleaching can occur due to the instability of the dyes associated with these constructs.

SWNTs have also been specifically targeted to tumors overexpressing the cell surface glycoprotein CD44 by coating them with a hyaluronic acid-based biosurfactant. The coating is degraded by hyaluronidases overexpressed in cancer cells. Further labeling of the hyaluronic acid-unit with a fluorescent dye was used to provide validation of the selective CD44 receptor uptake using PAI and fluorescence imaging ${ }^{83}$ illustrating controlled SWNT delivery.

Although CNPs are generally limited by their broadband absorption profile and low molar extinction coefficient (similar to dyes), they offer high photostability and versatile routes for functionalization and tailoring of spectral properties. Limited investigations of 
biocompatibility suggest that as with GNPs, surface modification, size, shape and synthesis method play a role. ${ }^{91}$ Several studies have reported cytotoxicity and inflammatory potential of SWNTs ${ }^{92,93}$, although this can be significantly reduced by functionalization. ${ }^{94,95}$ On the contrary, emerging evidence suggests that nanodiamonds exhibit high biocompatibility ${ }^{96,97,98}$. Significant future effort is needed to better understand CNP photophysics and potential for biocompatibility.

\section{Organic Nanostructures}

An exciting recent development in molecular PAI is the use of polymer nanoparticles and encapsulations (Supplementary Table 4). They have high structural and functional flexibility (composition, shape, charge, size) and can act directly as signaling compounds, enhance biocompatibility of other signaling compounds, or act as a foundation for activatable imaging probes in PAI. The most popular organic polymer structures for PAI contain either conjugated polymers or porphyrin related units (Supplementary Fig. 6), which directly absorb light. Their molar extinction coefficient sits between the small molecule dyes and GNPs (Fig. 4), and while their absorption spectra may be broad, they usually exhibit an absorption peak in the NIR (Fig. 7d) and are relatively photostable. Furthermore, their chemically and physically stable polymeric platform provides the opportunity to attach specific targeting and therapeutic moieties (see Supplementary Note 2).

Polymer nanoparticles come in many different flavors. Conjugated polymers (CP) are macromolecular structures with a highly delocalized conjugated backbone (Supplementary Fig. 6a). The conjugated system is spread through the entire polymer backbone and the polymers are densely packed into NPs yielding much higher extinction coefficient and photostability than small molecule dyes. The optical properties (strongly dependent on the conjugated core) can be tuned by: adopting different backbone structures; combining different conjugated polymers; and controlling aggregation and surface functionalization. ${ }^{99-}$ ${ }^{101}$ As an example, polypyrrole NPs are advantageous for PAI due to intense NIR absorption, high photothermal conversion efficiency and photostability, without acute toxicity. ${ }^{102}$ They can be well dispersed in aqueous solution ${ }^{102}$ but lack functional groups for surface modification. Alternative CPs that can be enriched with functional units are being developed, ${ }^{103,104}$ characterized also by an alternating electron donor-acceptor structure that shifts the absorption spectrum towards longer wavelengths (Supplementary Fig. 6b). Compared with SWNTs and GNRs these have been shown to yield 4-fold higher PAI signal in vivo on a per mass basis, but lower than the GNRs when compared on a molar basis. ${ }^{104}$ They can also act as 'smart' contrast agents (Section 'Emerging concepts in molecular PAl'), for example, for in vivo real-time imaging of reactive oxygen species. ${ }^{104}$

Alternatives to synthetic CPs are the naturally occurring porphyrins, aromatic macrocycles from heme and chlorophylls containing four pyrrolic units linked by methane (Supplementary Fig. 6a). While CPs often possess a broadband absorption profile, porphyrin NPs show a narrow absorption peak in the NIR, allowing a clearer identification in PAI (Fig. 7d). The chemical characteristics of the porphyrin macrocycle offers architectural flexibility, which permits tuning of the optical properties (Supplementary Fig. 6b). As with uncoated CPNPs, unmodified porphyrins are insoluble. Lovell et al overcame this challenge by conjugating porphyrin to a phospholipid, which resulted in self-assembled porphysomes with a bilayer structure. ${ }^{105}$ The porphyrin molecules inside the porphysome create J-aggregates, red-shifting the absorption peak (Supplementary Fig. 6b), and causing fluorescence 
quenching to yield a strong NIR PA signal. ${ }^{106,105}$ The porphyrin-lipid shell was also used for encapsulation of carboxyfluorescein, doxorubicin ${ }^{105}$ or fluorinated gases enabling multimodal PAI, ultrasound and fluorescence imaging. ${ }^{107,108}$ As natural products, poryphyrins and porphysomes would be expected to show high biocompatibility, but due to their relative immaturity as contrast agents, this has yet to be established in vivo.

Other polymers lacking intrinsic optical absorption but showing favorable biocompatibility can incorporate signaling compounds through micellar or vesicular structures, which can also be used for targeted delivery of compounds. Gold nanobeacons are formed from small GNPs (2-4 nm) trapped within synthetic or natural amphilines, forming NPs $60-260 \mathrm{~nm}$ in size. ${ }^{109,110}$ Advantages of such formulations include improved photostability, biocompatibility, signal intensity and targeting ability, relative to the signaling compound alone. Disadvantages include the presently limited knowledge of how polymer NP structure and local signaling compound concentration influences PA signal generation, as well as the larger size of the encapsulation.

Despite their early stage of development, polymers and encapsulations have been demonstrated in numerous passive targeting applications: imaging mouse brain vasculature $^{102,111}$ and tumor sentinel lymph node identification ${ }^{104,112,105}$ as well as primary tumor characterization ${ }^{113-115}$. Wang et al actively targeted folate receptor positive MCF-7 breast cancer xenografts with poly(D,L-lactide-co-glycolide) (PLGA) lipid NP containing ICG for PA signal and folic acid for targeting (Fig. 7e); the fabricated NPs showed good stability, high folate-receptor targeting efficiency and good PA signal in vitro and in vivo (Fig. 7f). ${ }^{116}$ Conjugation of polypyrrole NPs to the same target has also been demonstrated. ${ }^{103}$ Biocompatibility in the latter study was assessed only in cells in vitro; studies in vivo were limited to evaluating biodistribution, which showed the expected involvement of the reticuloendothelial system in contrast agent clearance. ${ }^{103}$

The stable polymeric platform provides exciting opportunities to attach not only active targeting moieties but also activatable functionalities for smart probes (Section 'Emerging concepts in molecular PAl'). Given that this class of contrast agents is relatively new, detailed biocompatibility studies are still lacking to prove their potential for in vivo application in molecular PAI. Further work to develop biocompatible platforms with even higher NIR absorption and multifunctional capability is expected to appear in the coming years.

\section{Emerging concepts in molecular PAI}

Smart (or 'activatable') contrast agents elicit a prescribed signal change, such as a shift in the peak absorption wavelength, in response to a biological stimulus of interest. As well as increasing the range of possible molecular imaging targets, a key advantage of smart contrast agents is that they minimize the influence of the background signal from endogenous chromophores, hence offering the potential for high sensitivity of detection. Their dynamic response also facilitates the study of processes such as enzymatic activity in real-time. Although relatively few examples exist so far for PAI, targeting proteases including matrix metalloprotease ${ }^{6}$ and furin ${ }^{32}$ has proven popular. In such 'smart' contrast agents, the protease substrate (a short peptide) links a quencher (BHQ3) with either a fluorophore $(\text { Alexa750) })^{6}$ or NP (CuS) $)^{117}$. PA images acquired at the peak wavelengths of the two absorbers both show strong signals before the contrast agent reaches the target. Inside the tumor microenvironment, proteases cleave the linker, leaving only one absorption peak after cleavage, yielding a highly specific imaging approach to quantify protease activity non- 
invasively. Undesirable toxicity in off-target tissues with high physiological protease levels has yet to be assessed.

Using multi-modality contrast agents it is possible to image the same target with two or more approaches (overcoming weaknesses of each modality) or to readout multiple targets, building a more complete picture of the underlying biochemistry. Molecular imaging modalities can differ in sensitivity up to 6 orders of magnitude ${ }^{118}$ requiring a difficult compromise to satisfy the concentration requirements for each modality. While PAI has been combined with $\mathrm{PET}^{74}$ and/or $\mathrm{MRI}^{119,120}$ for whole body imaging to determine contrast agent biocompatibility, its natural partners are ultrasound and fluorescence imaging due to the acoustic detection and optical excitation paths. Several PA and contrast enhanced ultrasound (CE-US) contrast agents are available. ${ }^{9,108}$ Small molecule dyes used for PAI are also fluorescent, which can facilitate ex vivo validation of contrast agent biodistribution in whole organs and tissue sections.

\section{Outlook: Promise and future challenges}

PAI is an exciting new modality that uses non-ionizing radiation to perform real-time imaging with high spatial resolution and penetration depth. A broad range of application areas for molecular PAI have been identified, including oncology, neuroscience and cardiovascular diseases; basic research studies and preliminary clinical trials have focused primarily thus far on detection, staging and therapeutic monitoring in cancer.

Despite great progress in molecular PAI contrast agent development in recent years, several challenges must be overcome in order to facilitate uptake into basic research in the life sciences. It is crucial that researchers recognize the need for standardized reporting of experimental approaches, for example, with regard to the measured photophysical properties. Publications should report in detail their calculations of extinction coefficients on both a 'per mass' and 'per mole' basis, along with independent measurements of both optical and photoacoustic absorption spectra as a function of concentration and solvent. These data have a major impact on biocompatibility, since detection sensitivity in vivo is intrinsically linked to administered dose. Systematic chemical studies to improve solubility, stability and specific targeting capability are needed across all contrast agent classes, along with the development of reproducible, cost effective and scalable chemical syntheses and purification methods.

With photophysical and chemical characteristics optimized, criteria related to targeting and biocompatibility must also be fulfilled. The large size of nanostructures relative to physiological barriers may limit their application to intravascular targets for molecular imaging applications beyond cancer. Methods for nanostructure self-assembly in vivo may overcome such limitations. To provide convincing evidence of active targeting, both intraand extra-vascular, studies must include both cellular controls (e.g. knockdowns of the biological target) and physiological controls (e.g. blocking with competing ligands). Although the nanostructured materials reviewed here are often claimed to be inert, biocompatibility appears to be strongly dependent on the nanostructure size, shape and synthesis method used in their preparation. In future studies, biological interactions, immunogenicity, stability, circulation and target tissue retention must be characterized for individual nanostructures to define their safety profile; relying on general literature from the nanostructure class is insufficient. Assessment of biocompatibility at both the cellular (in vitro) and organismal (in 
vivo) levels is needed, including blood sampling and tissue analysis to identify potential acute and chronic inflammatory and toxicity effects.

With biocompatibility concerns addressed, molecular PAI then has to achieve technical and biological validation before acceptance as a routine tool. Technical validation requires assessment of precision (repeatability / reproducibility) and accuracy (ability to relate the imaging signal to the abundance of chromophore). Although there have been significant efforts relating to accuracy of $\mathrm{PAI}^{121}$, there remains a paucity of data relating to precision.

Key to routine application of molecular PAI is biological validation of the sensitivity and specificity of the readout in relation to the target biological entity or process. Comparison between contrast agents would benefit from standardization (e.g. through the use of published recommendations ${ }^{122}$ ) with regard to the disease model, tumor sizes, time points and reported metrics. This review has also revealed the near ubiquitous use of simple animal models, including subcutaneous tumors. Immunodeficient nude mice are also used routinely, which avoid the impact of skin melanin on optical absorption and minimize interactions with the immune system. While these systems provide an acceptable proof-ofconcept, translation of findings into transgenic mouse models on immune competent backgrounds (e.g. C57BL/6) will ultimately be needed for widespread uptake of the technique in preclinical research.

The exogenous contrast agents described here are still in the early stages of exploration, mostly demonstrated only in single 'proof-of-concept' studies. Opposing the current trend towards vast increases in the number of different signaling compounds available, we believe that to maximize impact in preclinical molecular imaging, it is crucial to better understand the photophysical and biological properties of the promising classes that have already been reported, to enable their routine use. Lessons learned from other areas of molecular imaging indicate that the field may accelerate by focusing development and commercial efforts on contrast agents that prove successful in rigorous studies of biocompatibility and achieve biological validation using realistic animal models of disease.

The advantages of molecular PAI can be appreciated across diverse applications. Here, we have highlighted applications in: detection of angiogenic vasculature; evaluation heterogeneity in tumor cell receptor expression; and visualization of protease activity. Contrast agents that meet both the photophysical and biological requirements set out in this review are likely to emerge in the near future. Such agents could rapidly advance studies of targeted cancer therapies, perhaps even tracking immune cell infiltration and activation in tumor models at high resolution to advance our understanding of the mechanisms of action and resistance for immunotherapy.

\section{Acknowledgments}

We would like to thank J. Baumberg from the Department of Physics in Cambridge for helpful discussions on gold nanoparticle LSPR. This work was supported by: a CRUK Career Establishment Award grant no. C47594/A16267 (J.W. and S.E.B.); CRUK Core Funding C14303/A17197 (J.W. and S.E.B.); EU CIG FP7-PEOPLE-2013-CIG-630729 (J.W. and S.E.B.); the EPSRC-CRUK Cancer Imaging Centre in Cambridge and Manchester C197/A16465 (J.W. and S.E.B.); King's College London and University College London 
Comprehensive Cancer Imaging Centre Cancer Research UK \& Engineering and Physical Sciences Research Council, in association with the Medical Research Council and Department of Health, UK (P.B.) and European Union project FAMOS FP7 ICT, Contract 317744 (P.B.). J.W., P.B. and S.E.B. performed the literature review and wrote the paper. 


\section{References}

1. Ntziachristos, V. Going deeper than microscopy: the optical imaging frontier in biology. Nat. Methods 7, 603-614 (2010).

2. Placeholder citation for complementary review.

3. Beard, P. Biomedical photoacoustic imaging. Interface Focus 1, 602-31 (2011).

4. Taruttis, a., van Dam, G. M. \& Ntziachristos, V. Mesoscopic and Macroscopic Optoacoustic Imaging of Cancer. Cancer Res. 75, 1548-1560 (2015).

5. Zackrisson, S., van de Ven, S. M. W. Y. \& Gambhir, S. S. Light In and Sound Out: Emerging Translational Strategies for Photoacoustic Imaging. Cancer Res. 74, 979 1004 (2014).

6. Levi, J. et al. Molecular Photoacoustic Imaging of Follicular Thyroid Carcinoma. Clin. Cancer Res. 19, 1494-1502 (2013).

7. Li, P.-C. et al. In vivo photoacoustic molecular imaging with simultaneous multiple selective targeting using antibody-conjugated gold nanorods. Opt. Express 16, 18605-18615 (2008).

8. Laufer, J. et al. In vivo preclinical photoacoustic imaging of tumor vasculature development and therapy. J. Biomed. Opt. 17, 056016 (2012).

9. Luke, G. P., Myers, J. N., Emelianov, S. Y. \& Sokolov, K. V. Sentinel lymph node biopsy revisited: Ultrasound-guided photoacoustic detection of micrometastases using molecularly targeted plasmonic nanosensors. Cancer Res. 74, 5397-5408 (2014).

10. Bohndiek, S. et al. Photoacoustic tomography detects early vessel regression and normalization during ovarian tumor response to the anti-angiogenic therapy Trebananib. J. Nucl. Med. 215, (2015).

11. Guggenheim, J. a. et al. Photoacoustic imaging of human lymph nodes with endogenous lipid and hemoglobin contrast. J. Biomed. Opt. 20, 050504 (2015).

12. Xu, Z., Zhu, Q. \& Wang, L. V. In vivo photoacoustic tomography of mouse cerebral edema induced by cold injury. J. Biomed. Opt. 16, 066020 (2011).

13. Zhang, H. F., Maslov, K., Stoica, G. \& Wang, L. V. Functional photoacoustic microscopy for high-resolution and noninvasive in vivo imaging. Nat. Biotechnol. 24, 848-851 (2006).

14. Oh, J.-T. et al. Three-dimensional imaging of skin melanoma in vivo by dualwavelength photoacoustic microscopy. J. Biomed. Opt. 11, 34032-1 -34032-4 (2006).

15. Stoffels, I. et al. Metastatic status of sentinel lymph nodes in melanoma determined noninvasively with multispectral optoacoustic imaging. Sci. Transl. Med. 7, (2015).

16. Kang, J. H. \& Chung, J.-K. Molecular-Genetic Imaging Based on Reporter Gene Expression. J. Nucl. Med. 49, 164S-179S (2008).

17. Razansky, D. et al. Multispectral opto-acoustic tomography of deep-seated fluorescent proteins in vivo. Nat. Photonics 3, 412-417 (2009).

18. Laufer, J., Jathoul, A., Pule, M. \& Beard, P. In vitro characterization of genetically expressed absorbing proteins using photoacoustic spectroscopy. Biomed. Opt. Express 4, 2477-90 (2013).

19. Shu, X. et al. Mammalian Expression of Infrared Fluorescent Proteins Engineered from a Bacterial Phytochrome. Science (80-. ). 324, 804-807 (2009).

20. Filonov, G. S. et al. Deep-tissue photoacoustic tomography of a genetically encoded 
near-infrared fluorescent probe. Angew. Chemie - Int. Ed. 51, 1448-1451 (2012).

21. Yao, J. et al. Multiscale photoacoustic tomography using reversibly switchable bacterial phytochrome as a near-infrared photochromic probe. Nat. Methods 1-9 (2015). doi:10.1038/nmeth.3656

22. Cai, X. et al. Multi-scale molecular photoacoustic tomography of gene expression. PLoS One 7, 1-7 (2012).

23. Jathoul, A. P. et al. Deep in vivo photoacoustic imaging of mammalian tissues using a tyrosinase-based genetic reporter. Nat. Photonics 9, 239-246 (2015).

24. Qin, C. et al. Tyrosinase as a multifunctional reporter gene for Photoacoustic/MRI/PET triple modality molecular imaging. Sci. Rep. 3, 1490 (2013).

25. Urabe, K. et al. The inherent cytotoxicity of melanin precursors: a revision. Biochim. Biophys. Acta 1221, 272-278 (1994).

26. Tenzer, S. et al. Rapid formation of plasma protein corona critically affects nanoparticle pathophysiology. Nat. Nanotechnol. 8, 772-81 (2013).

27. Lynch, I. \& Dawson, K. a. Protein-nanoparticle interactions. Nano Today 3, 40-47 (2008).

28. Lundqvist, M. Nanoparticles: Tracking protein corona over time. Nat. Publ. Gr. 8, 1-2 (2013).

29. Petros, R. a. \& DeSimone, J. M. Strategies in the design of nanoparticles for therapeutic applications. Nat. Rev. Drug Discov. 9, 615-627 (2010).

30. Nie, L. et al. In vivo volumetric photoacoustic molecular angiography and therapeutic monitoring with targeted plasmonic nanostars. Small 10, 1585-1593 (2014).

31. Chatni, M. R. et al. Tumor glucose metabolism imaged in vivo in small animals with whole-body photoacoustic computed tomography. J. Biomed. Opt. 17, 076012 (2012).

32. Dragulescu-Andrasi, A., Kothapalli, S. R., Tikhomirov, G. a., Rao, J. \& Gambhir, S. S. Activatable oligomerizable imaging agents for photoacoustic imaging of furin-like activity in living subjects. J. Am. Chem. Soc. 135, 11015-11022 (2013).

33. Tiede, C. et al. Adhiron: A stable and versatile peptide display scaffold for molecular recognition applications. Protein Eng. Des. Sel. 27, 145-155 (2014).

34. Löfblom, J. et al. Affibody molecules: Engineered proteins for therapeutic, diagnostic and biotechnological applications. FEBS Lett. 584, 2670-2680 (2010).

35. Sun, H. et al. Oligonucleotide aptamers: new tools for targeted cancer therapy. Mol. Ther. Nucleic Acids 3, e182 (2014).

36. Olafsen, T. \& Wu, A. M. Antibody Vectors for Imaging. Semin. Nucl. Med. 40, 167181 (2010).

37. Matsumura, Y. \& Maeda, H. A new concept for macromolecular therapeutics in cancer chemotherapy: mechanism of tumoritropic accumulation of proteins and the antitumor agents Smancs. Cancer Res. 46, 6387- 6392 (1986).

38. Clark, A. J. et al. CRLX101 nanoparticles localize in human tumors and not in adjacent, nonneoplastic tissue after intravenous dosing. Proc. Natl. Acad. Sci. 113, 201603018 (2016).

39. Luo, S., Zhang, E., Su, Y., Cheng, T. \& Shi, C. A review of NIR dyes in cancer targeting and imaging. Biomaterials 32, 7127-7138 (2011).

40. Jaffe, H. H. \& Miller, A. L. The fates of electronic excitation energy. J. Chem. Educ. 43, 469-473 (1966). 
41. Lin, Y., Weissleder, R. \& Tung, C. H. Novel near-infrared cyanine fluorochromes: Synthesis, properties, and bioconjugation. Bioconjug. Chem. 13, 605-610 (2002).

42. Song, F. et al. Syntheses, spectral properties and photostabilities of novel watersoluble near-infrared cyanine dyes. J. Photochem. Photobiol. A Chem. 168, 53-57 (2004).

43. Philip, R., Penzkofer, a, Baumler, W., Szeimies, R. M. \& Abels, C. Absorption and fluorescence spectroscopic investigation of indocyanine green. J. Photochem. Photobiol. A-Chemistry 96, 137-148 (1996).

44. Kuebler, W. M. How NIR is the future in blood flow monitoring? J. Appl. Physiol. 104, 905-906 (2008).

45. Kim, C., Song, K. H., Gao, F. \& Wang, L. V. Sentinel Lymph Nodes and Lymphatic Vessels : Noninvasive Dual-Modality in Vivo Mapping by Using Indocyanine Green in Rats - Volumetric Spectroscopic Photoacoustic Imaging and Planar Fluorescence Imaging. Radiology 255, 442-450 (2010).

46. Landsman, M. L., Kwant, G., Mook, G. a \& Zijlstra, W. G. Light-absorbing properties, stability, and spectral stabilization of indocyanine green. J. Appl. Physiol. 40, 575-583 (1976).

47. Mordon, S., Devoisselle, J. M., Soulie-Begu, S. \& Desmettre, T. Indocyanine green: physicochemical factors affecting its fluorescence in vivo. Microvasc. Res. 55, 146152 (1998).

48. Pauli, J. et al. An in vitro characterization study of new near infrared dyes for molecular imaging. Eur. J. Med. Chem. 44, 3496-3503 (2009).

49. Umezawa, K., Citterio, D. \& Suzuki, K. Water-soluble NIR fluorescent probes based on squaraine and their application for protein labeling. Anal. Sci. 24, 213-217 (2008).

50. Sreejith, S., Carol, P., Chithra, P. \& Ajayaghosh, A. Squaraine dyes: a mine of molecular materials. J. Mater. Chem. 18, 264 (2008).

51. Kim, S. H. et al. Absorption spectra, aggregation and photofading behaviour of nearinfrared absorbing squarylium dyes containing perimidine moiety. Dye. Pigment. 55, 1-7 (2002).

52. Zhang, D. et al. Nano-Confined Squaraine Dye Assemblies: New Photoacoustic and Near-Infrared Fluorescence Dual-Modular Imaging Probes in Vivo. Bioconjug. Chem. 25, $2021-2029$ (2014).

53. An, F. et al. Aggregation-Induced Near-Infrared Absorption of Squaraine Dye in an Albumin Nanocomplex for Photoacoustic Tomography in Vivo. Appl. Mater. Interfaces (2014).

54. Sreejith, S., Joseph, J., Lin, M., Menon, N. V. \& Borah, P. Near-Infrared Squaraine Dye Encapsulated Micelles for in Vivo Fluorescence and Photoacoustic Bimodal Imaging. ACS Nano 9, 5695-5704 (2015).

55. Beija, M., Afonso, C. a M. \& Martinho, J. M. G. Synthesis and applications of Rhodamine derivatives as fluorescent probes. Chem. Soc. Rev. 38, 2410-2433 (2009).

56. Morgounova, E., Shao, Q., Hackel, B. J., Thomas, D. D. \& Ashkenazi, S.

Photoacoustic lifetime contrast between methylene blue monomers and selfquenched dimers as a model for dual-labeled activatable probes. J. Biomed. Opt. 18, 56004 (2013).

57. Yao, J., Maslov, K., Hu, S. \& Wang, L. V. Evans blue dye-enhanced capillaryresolution photoacoustic microscopy in vivo. J. Biomed. Opt. 14, 054049 (2009). 
58. Gabrielli, D., Belisle, E., Severino, D., Kowaltowski, A. J. \& Baptista, M. S. Binding, aggregation and photochemical properties of methylene blue in mitochondrial suspensions. Photochem. Photobiol. 79, 227-232 (2004).

59. Onoe, S., Temma, T., Kanazaki, K., Ono, M. \& Saji, H. Development of photostabilized asymmetrical cyanine dyes for in vivo photoacoustic imaging of tumors Development of photostabilized asymmetrical cyanine dyes for in vivo photoacoustic imaging of tumors. J. Biomed. Opt. 20, 096006 (2015).

60. Reynolds, G., Reynolds, G., Drexhage, K. \& Drexhage, K. Stable heptamethine pyrylium dyes that absorb in the infrared. J. Org. Chem. 42, 885-888 (1977).

61. Hudson, S. V. et al. Targeted Noninvasive Imaging of EGFR-Expressing Orthotopic Pancreatic Cancer Using Multispectral Optoacoustic Tomography. Cancer Res. 74, 6271-6279 (2014).

62. Duncan, R. \& Gaspar, R. Nanomedicine(s) under the microscope. Mol. Pharm. 8, 2101-2141 (2011).

63. Daniel, M. C. \& Astruc, D. Gold Nanoparticles: Assembly, Supramolecular Chemistry, Quantum-Size-Related Properties, and Applications Toward Biology, Catalysis, and Nanotechnology. Chem. Rev. 104, 293-346 (2004).

64. Chen, Y.-S. et al. Enhanced thermal stability of silica-coated gold nanorods for photoacoustic imaging and image-guided therapy. Opt. Express 18, 8867-8878 (2010).

65. Willets, K. a \& Van Duyne, R. P. Localized surface plasmon resonance spectroscopy and sensing. Annu. Rev. Phys. Chem. 58, 267-297 (2007).

66. Petryayeva, E. \& Krull, U. J. Localized surface plasmon resonance: Nanostructures, bioassays and biosensing-A review. Anal. Chim. Acta 706, 8-24 (2011).

67. Chen, Y. S. et al. Silica-coated gold nanorods as photoacoustic signal nanoamplifiers. Nano Lett. 11, 348-354 (2011).

68. Cavigli, L. et al. Size Affects the Stability of the Photoacoustic Conversion of Gold Nanorods. J. Phys. Chem. 118, 16140-16146 (2014).

69. Preston, T. C. \& Signorell, R. Growth and optical properties of gold nanoshells prior to the formation of a continuous metallic layer. ACS Nano 3, 3696-3706 (2009).

70. Skrabalak, S. E. et al. Gold Nanocages: Synthesis, Properties and Applications. Acc. Chem. Res. 41, 1587-1595 (2008).

71. Srivatsan, A. et al. Gold nanocage-photosensitizer conjugates for dual-modal imageguided enhanced photodynamic therapy. Theranostics 4, 163-174 (2014).

72. Xie, H. et al. Integrin avß3-targeted gold nanoshells augment tumor vasculaturespecific imaging and therapy. Int. J. Nanomedicine 6, 259-269 (2011).

73. Fard, J. K., Jafari, S. \& Eghbal, M. A. A Review of Molecular Mechanisms Involved in Toxicity of Nanoparticles. Adv. Pharm. Bullrtin 5, 447-454 (2015).

74. Cheng, K. et al. Construction and validation of nano gold tripods for molecular imaging of living subjects. J. Am. Chem. Soc. 136, 3560-3571 (2014).

75. Zhang, M., Yudasaka, M., Ajima, K., Miyawaki, J. \& lijima, S. Light-assisted oxidation of single-wall carbon nanohorns for abundant creation of oxygenated groups that enable Chemical modifications with proteins to enhance biocompatibility. ACS Nano 1, 265-272 (2007).

76. Krueger, A. \& Lang, D. Functionality is key: Recent progress in the surface modification of nanodiamond. Adv. Funct. Mater. 22, 890-906 (2012). 
77. He, H. \& Gao, C. General approach to individually dispersed, highly soluble, and conductive graphene nanosheets functionalized by nitrene chemistry. Chem. Mater. 22, 5054-5064 (2010).

78. Bahr, J. L. et al. Functionalization of carbon nanotubes by electrochemical reduction of aryl diazonium salts: A bucky paper electrode. J. Am. Chem. Soc. 123, 6536-6542 (2001).

79. De La Zerda, A. et al. Family of enhanced photoacoustic imaging agents for highsensitivity and multiplexing studies in living mice. ACS Nano 6, 4694-4701 (2012).

80. Liu, Z., Robinson, J. T., Sun, X. \& Dai, H. PEGylated Nano-Graphene Oxide for Delivery of Water Insoluble Cancer Drugs (b). J Am Chem Soc 130, 10876-10877 (2008).

81. O'Connell, M. J. et al. Band gap fluorescence from individual single-walled carbon nanotubes. Science 297, 593-596 (2002).

82. Shao, Y. et al. Graphene based electrochemical sensors and biosensors: A review. Electroanalysis 22, 1027-1036 (2010).

83. Swierczewska, M. et al. A Facile, One-Step Nanocarbon Functionalization for Biomedical Applications. Nano Lett. 12, 3613-3620 (2012).

84. Vial, S. et al. Peptide-grafted nanodiamonds: Preparation, cytotoxicity and uptake in cells. ChemBioChem 9, 2113-2119 (2008).

85. Avouris, P., Freitag, M. \& Perebeinos, V. Carbon-nanotube photonics and optoelectronics. Nat. Photonics 2, 341-350 (2008).

86. Carlson, L. J. \& Krauss, T. D. Photophysics of individual single-walled carbon nanotubes. Acc. Chem. Res. 41, 235-243 (2008).

87. Bachilo, S. M. et al. Structure-Assigned Optical Spectra of Single-Walled Carbon Nanotubes. Science (80-. ). 298, 2361-2366 (2002).

88. De la Zerda, A. et al. Carbon nanotubes as photoacoustic molecular imaging agents in living mice. Nat. Nanotechnol. 3, 557-562 (2008).

89. Wang, C. et al. RGD-conjugated silica-coated gold nanorods on the surface of carbon nanotubes for targeted photoacoustic imaging of gastric cancer. Nanoscale Reserach Lett. 9, 1-10 (2014).

90. Zerda, A. D. La et al. Ultrahigh sensitivity carbon nanotube agents for photoacoustic molecular imaging in living mice. Nano Lett. 10, 2168-2172 (2010).

91. Seabra, A. B., Paula, A. J., De Lima, R., Alves, O. L. \& Durán, N. Nanotoxicity of graphene and graphene oxide. Chem. Res. Toxicol. 27, 159-168 (2014).

92. Kostarelos, K. The long and short of carbon nanotube toxicity. Nat. Biotechnol. 26, 774-776 (2008).

93. Wick, P., Clift, M. J. D., Rösslein, M. \& Rothen-Rutishauser, B. A brief summary of carbon nanotubes science and technology: A health and safety perspective. ChemSusChem 4, 905-911 (2011).

94. Schipper, M. L. et al. A pilot toxicology study of single-walled carbon nanotubes in a small sample of mice. Nat. Nanotechnol. 3, 216-221 (2008).

95. Saito, N. et al. Safe clinical use of carbon nanotubes as innovative biomaterials. Chem. Rev. 114, 6040-6079 (2014).

96. Kaur, R. \& Badea, I. Nanodiamonds as novel nanomaterials for biomedical applications: Drug delivery and imaging systems. Int. J. Nanomedicine 8, 203-220 (2013). 
97. Mochalin, V. N., Shenderova, O., Ho, D. \& Gogotsi, Y. The properties and applications of nanodiamonds. Nat. Nanotechnol. 7, 11-23 (2011).

98. Zhu, Y. et al. The biocompatibility of nanodiamonds and their application in drug delivery systems. Theranostics 2, 302-312 (2012).

99. Nguyen, T.-Q., Martini, I. B., Liu, J. \& Schwartz, B. J. Controlling Interchain Interactions in Conjugated Polymers: The Effects of Chain Morphology on Exciton-Exciton Annihilation and Aggregation in MEH-PPV Films. J. Phys. Chem. B 104, 237-255 (2000).

100. Feng, L. et al. Conjugated polymer nanoparticles: preparation, properties, functionalization and biological applications. Chem. Soc. Rev. 42, 6620-33 (2013).

101. Wu, C. et al. Bioconjugation of ultrabright semiconducting polymer dots for specific cellular targeting. J. Am. Chem. Soc. 132, 15410-15417 (2010).

102. Zha, Z. et al. Biocompatible polypyrrole nanoparticles as a novel organic photoacoustic contrast agent for deep tissue imaging. Nanoscale 5, 4462-7 (2013).

103. Balasundaram, G. et al. Molecular photoacoustic imaging of breast cancer using an actively targeted conjugated polymer. Int. J. Nanomedicine 10, 387-397 (2015).

104. Pu, K. et al. Semiconducting polymer nanoparticles as photoacoustic molecular imaging probes in living mice. Nat. Nanotechnol. 9, 233-9 (2014).

105. Lovell, J. F. et al. Porphysome nanovesicles generated by porphyrin bilayers for use as multimodal biophotonic contrast agents. Nat. Mater. 10, 324-332 (2011).

106. Ng, K. K. et al. Stimuli-Responsive Photoacoustic Nanoswitch for In Vivo Sensing Applications. ACS Nano 8, 8363-8373 (2014).

107. Huynh, E., Jin, C. S., Wilson, B. C. \& Zheng, G. Aggregate enhanced trimodal porphyrin shell microbubbles for ultrasound, photoacoustic, and fluorescence imaging. Bioconjug. Chem. 25, 796-801 (2014).

108. Huynh, E. et al. In situ conversion of porphyrin microbubbles to nanoparticles for multimodality imaging. Nat. Nanotechnol. 10, 325-332 (2015).

109. Pan, D. et al. Molecular photoacoustic tomography with colloidal nanobeacons. Angew. Chemie - Int. Ed. 48, 4170-4173 (2009).

110. Pan, D., Pramanik, M., Wickline, S. a., Wang, L. V. \& Lanza, G. M. Recent advances in colloidal gold nanobeacons for molecular photoacoustic imaging. Contrast Media Mol. Imaging 6, 378-388 (2011).

111. Liu, J. et al. Conjugated polymer nanoparticles for photoacoustic vascular imaging. Polym. Chem. 5, 2854 (2014).

112. Pan, D. et al. Rapid synthesis of near infrared polymeric micelles for real-time sentinel lymph node imaging. Adv. Healthc. Mater. 1, 582-589 (2012).

113. Jokerst, J. V., Van de Sompel, D., Bohndiek, S. E. \& Gambhir, S. S. Cellulose nanoparticles are a biodegradable photoacoustic contrast agent for use in living mice. Photoacoustics 2, 119-127 (2014).

114. Miki, K. et al. Near-Infrared Dye-Conjugated Amphiphilic Hyaluronic Acid Derivatives as a Dual Contrast Agent for In Vivo Optical and Photoacoustic Tumor Imaging. Biomacromolecules 16, 219-227 (2015).

115. Aoki, H., Nojiri, M., Mukai, R. \& Ito, S. Near-infrared absorbing polymer nano-particle as a sensitive contrast agent for photo-acoustic imaging. Nanoscale 7, 337-343 (2015).

116. Wang, $\mathrm{H}$. et al. In vivo photoacoustic molecular imaging of breast carcinoma with 
folate receptor-targeted indocyanine green nanoprobes. Nanoscale 6, 14270-14279 (2014).

117. Yang, K. et al. Visualization of protease activity in vivo using an activatable photoacoustic imaging probe based on CuS nanoparticles. Theranostics 4, 134-141 (2014).

118. Massoud, T. F. \& Gambhir, S. S. Molecular imaging in living subjects: seeing fundamental biological processes in a new light. Genes Dev. 17, 545-580 (2003).

119. Hembury, M. et al. Gold-silica quantum rattles for multimodal imaging and therapy. Proc. Natl. Acad. Sci. 112, 1959-1964 (2015).

120. Gong, $\mathrm{H}$. et al. Engineering of multifunctional nano-micelles for combined photothermal and photodynamic therapy under the guidance of multimodal imaging. Adv. Funct. Mater. 1-11 (2014). doi:10.1002/adfm.201401451

121. Cox, B., Laufer, J. G., Arridge, S. R. \& Beard, P. C. Quantitative spectroscopic photoacoustic imaging: a review. J. Biomed. Opt. 17, 061202 (2012).

122. Dawidczyk, C. M., Russell, L. M. \& Searson, P. C. Recommendations for Benchmarking Preclinical Studies of Nanomedicines. Cancer Res. 75, 4016-4020 (2015).

123. Hale, G. M. \& Querry, M. R. Optical Constants of Water in the 200-nm to 200-microm Wavelength Region. Appl. Opt. 12, 555-563 (1973).

124. Veen, R. L. P. van et al. Determination of visible near-IR absorption coefficients of mammalian fat using time- and spatially resolved diffuse reflectance and transmission spectroscopy. J. Biomed. Opt. 10, 054004 (2005).

125. Tsai, C. L., Chen, J. C. \& Wang, W. J. Near-infrared absorption property of biological soft tissue constituents. J. Med. Biol. Eng. 21, 7-14 (2001).

126. Lozano, N., Al-ahmady, Z. S., Beziere, N. S., Ntziachristos, V. \& Kostarelos, K. Monoclonal antibody-targeted PEGylated liposome-ICG encapsulating doxorubicin as a potential theranostic agent. Int. J. Pharm. (2014). doi:10.1016/j.jpharm.2014.10.045

127. Eghtedari, M. et al. Engineering of hetero-functional gold nanorods for the in vivo molecular targeting of breast cancer cells. Nano Lett. 9, 287-91 (2009).

128. Ashkenazi, S. Photoacoustic lifetime imaging of dissolved oxygen using methylene blue. J. Biomed. Opt. 15, 040501 (2010).

129. Onoe, S., Temma, T., Shimizu, Y., Ono, M. \& Saji, H. Investigation of cyanine dyes for in vivo optical imaging of altered mitochondrial membrane potential in tumors. Cancer Med. 3, 775-86 (2014).

130. Kang, N.-Y. et al. A macrophage uptaking near-infrared chemical probe for in vivo imaging of inflammation. Chem. Commun. (Camb). 50, 6589-91 (2014).

131. Beziere, N. \& Ntziachristos, V. Optoacoustic Imaging of Naphthalocyanine: Potential for Contrast Enhancement and Therapy Monitoring. J. Nucl. Med. 56, 323-328 (2014).

132. Liu, X., Atwater, M., Wang, J. \& Huo, Q. Extinction coefficient of gold nanoparticles with different sizes and different capping ligands. Colloids Surf. B. Biointerfaces 58, 3-7 (2007).

133. de Puig, H., Tam, J. O., Yen, C.-W., Gehrke, L. \& Hamad-Schifferli, K. Extinction Coefficient of Gold Nanostars. J. Phys. Chem. C 119, 17408-17415 (2015).

134. Jokerst, J. V., Cole, A. J., Van De Sompel, D. \& Gambhir, S. S. Gold nanorods for ovarian cancer detection with photoacoustic imaging and resection guidance via 
Raman imaging in living mice. ACS Nano 6, 10366-10377 (2012).

135. Beqa, L., Fan, Z., Singh, A. K., Senapati, D. \& Ray, P. C. Gold nano-popcorn attached SWCNT hybrid nanomaterial for targeted diagnosis and photothermal therapy of human breast cancer cells. ACS Appl. Mater. Interfaces 3, 3316-3324 (2011).

136. Leonov, A. P. et al. Detoxification of gold nanorods by treatment with polystyrenesulfonate. ACS Nano 2, 2481-2488 (2008).

137. Hu, J. et al. Theranostic Au cubic nano-aggregates as potential photoacoustic contrast and photothermal therapeutic agents. Theranostics 4, 534-545 (2014).

138. Lu, W. et al. Effects of photoacoustic imaging and photothermal ablation therapy mediated by targeted hollow gold nanospheres in an orthotopic mouse xenograft model of glioma. Cancer Res. 71, 6116-6121 (2011).

139. Clavero, C. Plasmon-induced hot-electron generation at nanoparticle/metal-oxide interfaces for photovoltaic and photocatalytic devices. Nat. Photonics 8, 95-103 (2014).

140. Brongersma, M. L., Halas, N. J. \& Nordlander, P. Plasmon-induced hot carrier science and technology. Nat. Publ. Gr. 10, 25-34 (2015).

141. Li, J., Guo, H. \& Li, Z. Microscopic and macroscopic manipulation of gold nanorod and its hybrid nanostructures. Photonics Res. 1, 28-41 (2013).

142. Jain, P. K. \& El-Sayed, M. a. Universal scaling of plasmon coupling in metal nanostructures: extension from particle pairs to nanoshells. Nano Lett. 7, 2854-8 (2007).

143. Hodge, S. a., Bayazit, M. K., Coleman, K. S. \& Shaffer, M. S. P. Unweaving the rainbow: a review of the relationship between single-walled carbon nanotube molecular structures and their chemical reactivity. Chem. Soc. Rev. 41, 4409 (2012).

144. Zerda, A. D. La et al. Ultrahigh sensitivity carbon nanotube agents for photoacoustic molecular imaging in living mice. Nano Lett. 10, 2168-2172 (2010). 


\section{Figure Legends for Main Text}

Figure 1. Photoacoustic imaging of endogenous chromophores. (a) Absorption spectra of the main endogenous chromophores: Oxyhemoglobin $\left(\mathrm{HbO}_{2}\right)$, red line $\left(150 \mathrm{gl}^{-1}\right)$; deoxyhemoglobin $(\mathrm{Hb})$, blue line $\left(150 \mathrm{gl}^{-1}\right)$; water, black line ${ }^{123}(80 \%$ by volume in tissue); lipid, yellow lines ${ }^{124,125}$ ( $20 \%$ by volume in tissue); melanin, brown line $\left(\mu_{\mathrm{a}}\right.$ corresponds to that in skin); collagen, green line. Data from http://omlc.ogi.edu/spectra/. (b) The heme group is a strongly-absorbing iron containing aromatic porphyrin ring system that exists in different configurations before and after oxygen binding, which produce characteristic changes in the absorption spectrum. (c) PAI $(\lambda=640 \mathrm{~nm})$ of tumor vasculature showing the effect of the vascular disrupting agent OXi4503. ${ }^{8}$

Figure 2. Photoacoustic imaging of genetically encoded chromophores. (a) Simplified schematic demonstration of a general enzymatic reporter gene construct. (b) Photoswitching chemistry of Biliverdin from the Pfr state to the Pr state, and vice versa, induced by NIR $(\sim 730-790 \mathrm{~nm})$ and far-red $(\sim 630-690 \mathrm{~nm})$ light respectively. ${ }^{21}$ (c) Deep PA computed tomography of a mouse U87 brain tumor expressing BphP1, with the surrounding vasculature show in grayscale. The tumor (in color) was $\sim 3 \mathrm{~mm}$ beneath the scalp surface. ${ }^{21}$ (d) Retroviral vector construct for stable transduction of cells for visualization of tyrosinase (Tyr) expression mammalian cells. (LTR: long terminal repeat, includes the promotor; SD: splice donor; $\psi$ : viral packaging signal, which directs the incorporation of vector RNA into virions; SA: splice acceptor; IRES: internal ribosome entry sites). ${ }^{23}$ (e) Serial longitudinal in vivo PA images of stably transduced Tyr-expressing K562 cells after subcutaneous injection into the flank of a nude mouse, illustrating sensitive detection of produced melanin with depth resolution afforded by PAI (spatial resolution $<100 \mu \mathrm{m}$ at up to $10 \mathrm{~mm}$ depth). ${ }^{23}$

Figure 3. Assembly of molecular imaging contrast agents for PAl combining a signaling compound with a targeting ligand. Current strategies for the development of targeted contrast agents, are: the direct conjugation of signaling compounds with targeting ligands; encapsulation of signaling compounds (and targeting ligand) by nanostructures; or encapsulation (with targeting ligands) and synthesis of target-activatable signaling compounds. The targeting ligand and signaling compound should be compatible in their chemical, physical and biological properties. Examples of the relative advantages and disadvantages of each approach are indicated by +/-. ${ }^{31,32,120,126,127}$

Figure 4. Reported extinction coefficients of a selection of signaling compounds used for PAI. References for reported values are: peptide-grafted nanodiamonds ${ }^{84}$; methylene blue $^{128} ; \quad$ IC7-1-Bu ${ }^{129}$; CDnir7 ${ }^{130}$; IRDye800CW, ICG (http://www.spectra.arizona.edu/); $\mathrm{SiNc}^{131}$; gold nanospheres ${ }^{132}$; SWNTs ${ }^{104}$; SPN1 ${ }^{104}$; gold nanostar ${ }^{133}$; porphysome ${ }^{105}$; gold nanorod $^{134}$; gold nanopopcorn ${ }^{135}$; conjugated polymer $\mathrm{NP}^{104}$; cellulose $\mathrm{NP}^{113}$; gold nanocages $^{71}$; PSS-coated gold nanorods ${ }^{136}$; gold nanocubes ${ }^{137}$; and gold nanoshells ${ }^{138} \cdot \lambda$ represents the peak absorption wavelength.

Figure 5. Optical properties of near infrared (NIR) small molecule dyes and application for imaging the extravascular target epidermal growth factor receptor (EGFR) in pancreatic cancer. (a) General Jablonski diagram showing energetic transitions for an NIR dye after optical excitation. (b) Example absorption spectra of indocyanine green (ICG) in water and plasma in different concentrations, showing the significant impact of environmental conditions on spectral properties of NIR dyes, which can be challenging for PAI (spectra data used from University of Arizona http://www.spectra.arizona.edu/). (c) Multispectral optoacoustic tomography (MSOT) of mouse S2VP10L pancreatic adenocarcinoma at 7 days post implantation using EGF-CF-750 at 6 hours post-injection (Courtesy: Prof. L McNally). 
Figure 6. Optical properties of gold nanoparticles (GNP) and application for imaging the intravascular target $\alpha_{v} \beta_{3}$ integrin in glioma. (a) Localized surface plasmon resonance (LSPR) of spherical GNP and GNRs: electromagnetic waves induce plasmons, which can decay radiatively via re-emitting photons (scattering) or non-radiatively (essential for PAl). ${ }^{139,140}$ (b) Surface plasmon absorption spectra of gold nanorods of different aspect ratios, showing the sensitivity of the strong longitudinal band to the aspect ratios of the nanorods ( ILPSR = longitudinal LSPR; tLSPR = transverse LSPR). In comparison to GNR, gold nanospheres (grey line) show one absorption peak in green spectral range ${ }^{141}$ (c) Surface plasmon resonance frequency of gold nanoshells, tuned to the NIR range by varying the shell thickness relative to the core size. ${ }^{142}$ (d) PAI and ultrasound imaging of U87MG tumors in nude mice with intravenously injected $\alpha_{v} \beta_{3}$ integrin targeted gold tripods. ${ }^{74}$ (e) Chemical structure and UV-Vis spectroscopic profile $\alpha_{v} \beta_{3}$ integrin targeted gold tripods. ${ }^{74}$

Figure 7. Assembly and optical properties of single walled carbon nanotubes (SWNTs) and conjugated polymer nanoparticles (CPNPs), with applications in molecular imaging. (a) Schematic honeycomb structure of a graphene sheet, which can be rolled up from lattice point $(0,0)$ to $(n, m)$ along a chiral (roll-up) vector to form SWNT; the chiral vector length indicates the circumference/diameter of the tube and the chiral angle $(\alpha)$ the tilt between the hexagonal lattice and that vector. Depending on the diameter and twist the SWNT can be semiconducting (zigzag, blue $n, m$ numbers) or metallic (armchair, red $n, m$ numbers). Schematic illustration of the density of states (DOS) for semiconducting SWNT. (k = wavevector, $S_{11 / 22}=$ first/second energy transition). (b) UV-vis-NIR absorption spectra of SDS-suspended HiPco SWNT showing $S_{11}$ and $S_{22}$ regions. Resolved peaks correspond to various $(n, m)$ structures. ${ }^{143}$ (c) Difference images $(2 \mathrm{~h}$ postinjection - pre-scan) for ICGlabeled SWNT targeting U87MG glioma tumors. ${ }^{144}$ (d) Schematic of hybrid nanoparticles with a lipid monolayer shell and biodegradable polymer core (PLGA), which contain ICG for PA signal and DSPE-PEG-FA for targeting of folic acid (FA). (e) Absorption spectra of: a targeted conjugated polymer nanoparticle (CPNP, green line, structure shown in b and c) ${ }^{103}$; porphyrin based NPs encapsulating perfluorocarbon gas (red line) ${ }^{108}$; FA-ICG-PLGA-lipid polymer nanoparticle (dark blue line) ${ }^{116}$; and ICG (light blue, dashed line). (f) Imaging of MCF7 breast tumors using hybrid FA-ICG-PLGA-lipid nanoparticles shows tumor uptake at 24 hours post injection through the EPR effect (left), which is further enhanced by folic acid targeting (right). ${ }^{11}$

\section{Competing Financial Interests Statement}

The authors have no competing financial interests to declare. 